\title{
Dynamical Scattering and Protein Reconstruction by Electron Crystallography of Multi-layered Crystals
}

Oliver Vossen and Rasmus R. Schröder

Max-Planck-Institute for Medical Research, Jahnstr.29, D-69120 Heidelberg, Germany

Electron crystallography of biological samples has been used for reconstruction of several proteins [1]. The high scattering cross section of the electrons as opposed to photons in X-ray crystallography allows the use of smaller crystals. However, this high cross section also leads to dynamical scattering effects, which increase with the thickness of the crystal. Due to this limitation and the difficulty associated with interpreting data from thin 3D crystals, electron crystallography has so far been limited to 2D mono-layered crystals. If multi-layered crystals are to be used for structure determination, dynamical scattering must be understood. The effect of dynamical scattering on the usable resolution of the final reconstruction depends on the stacking of the protein in the crystal, the tilt angles selected, and on the electron energy used. It has been shown previously [2] that favourable stacking and carefully selected tilt angles can lead to reconstructions of samples up to $95 \mathrm{~nm}$. It is shown here that symmetrization of the diffraction data serves as a first order correction of dynamical scattering.

The multi-slice simulation software YaMS [3] was used to simulate diffraction patterns and images of 12 different proteins utilizing the atomic coordinates found in the PDB data base. Using the amplitude data from the diffraction pattern and the phase data from the images, reconstructions of the proteins were calculated. This was done for a mono-layered and for multi-layered samples consisting of several layers. To obtain a non-complex reconstruction, diffraction pattern amplitudes were symmetrized by averaging each Friedel pair. To illustrate the influence of dynamical scattering, the scattering data and the final reconstruction of one specific protein (bacteriorhodopsin) was analysed in detail. Assuming that data collected from a mono-layer sample is quasi-kinematical, a comparison with the data from 7 layers shows dynamical scattering effects. Figure 2 shows phase data for 1 and 7 layer simulations. Despite an overall similarity of the patterns, a detailed analysis shows considerable deviation (see Table 1). Note also that the magnitude of the deviation depends strongly on resolution. To assess the effect of dynamical scattering on the reconstruction, Fourier ring correlation coefficients between the 1 and 7 layer reconstructions were calculated and averaged for the 12 proteins. Figure 3 (solid lines) shows the Fourier ring correlation of two reconstructions, one at $120 \mathrm{keV}$ and $200 \mathrm{keV}$, respectively. Electrons at $200 \mathrm{keV}$ have a longer mean free path compared to lower energies and thus are less inclined to scatter dynamically. The correlation is thus slightly better than in the $120 \mathrm{keV}$ case.

Symmetrization of the diffraction pattern serves as a first order correction of the amplitude data. For the phase data the imaging process itself serves as a correction mechanism. The phase data taken from the image is anti-symmetrical since the simulated image is real. The dashed lines in figure 3 show reconstruction data from only half of the diffraction pattern i.e. unsymmetrized data. Clearly the strong dynamical scattering that is evident in the $\mathrm{R}_{\mathrm{sym}}$-factor [4] does not influence reconstructions of the data. A possible reconstruction scheme using phase data gathered from isomorphous replacement techniques [5] would be only marginally susceptible to dynamical scattering effects, since both the phase and the amplitude data could be taken from symmetrized diffraction patterns. 
[1] e.g. Henderson et al., JMB 213 (1989) p. 899-929

[2] Vossen \& Schroeder, Proceedings EUREM 12, Brno

[3] Dinges et al., Ultramicroscopy 67 (1995) p.207-217

[4] Glaeser \& Ceska , Acta Cryst. A45 (1989) p. 620-628

[5] Burmester \& Schroeder, Scanning Microscopy Supp. 11 (1997)

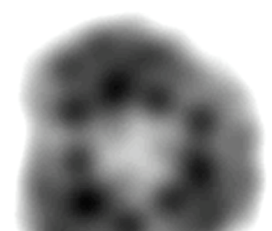

1 layer $=6.3 \mathrm{~nm}$

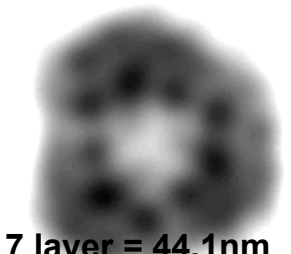

7 layer $=44.1 \mathrm{~nm}$

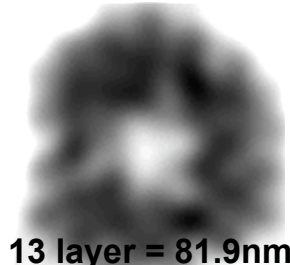

13 layer $=81.9 \mathrm{~nm}$

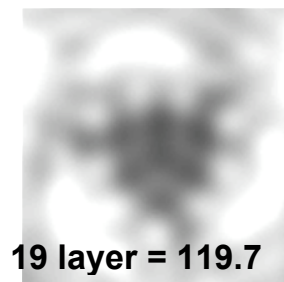

Figure 1: projections of simulated 3D reconstructions of bacteriorhodopsin at various crystal thicknesses. Note the appearance of the projected alpha helices.
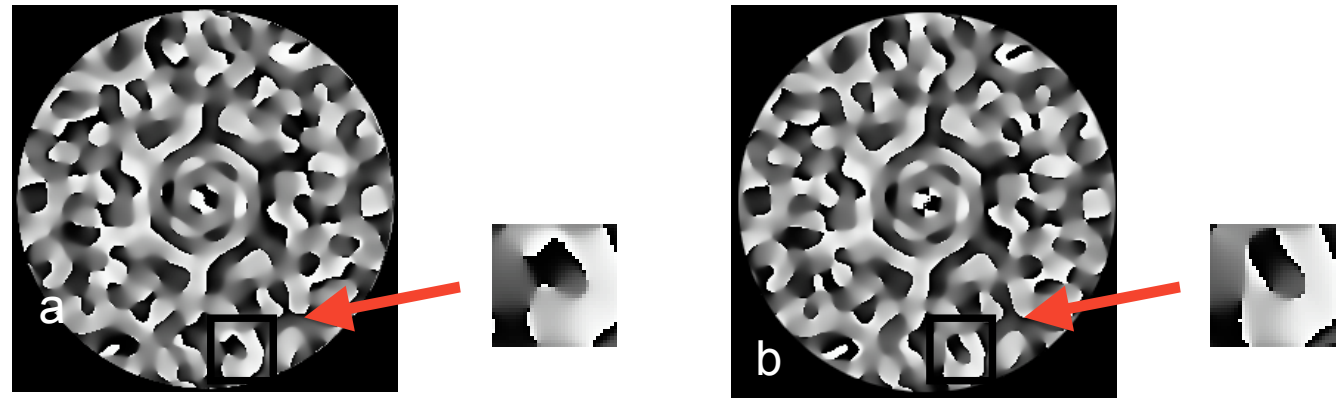

Figure 2: simulated Phase data from (a) 1 layer and (b) 7 layers of bacteriorhodopsin

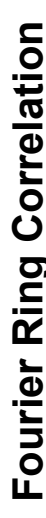

$$
1
$$

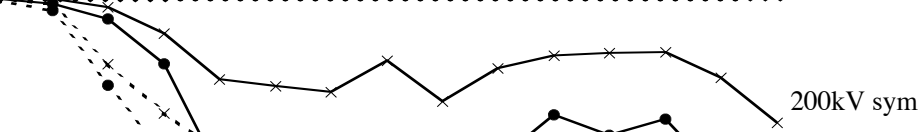

Figure 3: The Fourier Ring Correlation of the 1 and 7 layer reconstruction (averaged for 12 proteins) for 2 different electron energies (solid lines). The dashed lines show unsymmetrized data for comparison.

Resolution [§̊]

8 I

Table 1: Difference between 1 and 7 layer scattering data for phase and amplitude at different spatial resolutions. Note the small mean phase error and the reduced relative change of amplitude after symmetrization.

\begin{tabular}{l|c|c|c|c} 
& $\mathbf{1 6} \AA$ & $\mathbf{8 \AA}$ & $\mathbf{4 \AA}$ & $\mathbf{2 \AA}$ \\
\hline $\mathrm{I}\left[\left[^{\circ}{ }^{\circ}\right]\right.$ & 2.9 & 6.6 & 6.8 & 16.0 \\
$\square \mathrm{A} / \mathrm{A}[\%]$ (sym.) & 2.6 & 8.9 & 14.7 & 24.9 \\
$\mathrm{\square A} / \mathrm{A}[\%]$ (not sym.) & 4.9 & 20.1 & 28.0 & 39.3 \\
\# of Friedel pairs & 13 & 47 & 177 & 710 \\
\hline
\end{tabular}

